\title{
Lapurdum
}

Euskal ikerketen aldizkaria | Revue d'études basques |

Revista de estudios vascos | Basque studies review

$1 \mid 1996$

Numéro I

\section{Institutions, société et noblesse en pays basque : quelques textes significatifs}

Jean Goyhenetche

\section{(2) OpenEdition}

\section{Journals}

Édition électronique

URL : http://journals.openedition.org/lapurdum/1896

DOI : 10.4000/lapurdum.1896

ISSN : 1965-0655

Éditeur

IKER

Édition imprimée

Date de publication : 1 octobre 1996

Pagination : 171-189

ISBN : 2-84127-106-4

ISSN : $1273-3830$

Référence électronique

Jean Goyhenetche, «Institutions, société et noblesse en pays basque : quelques textes significatifs », Lapurdum [En ligne], 1 | 1996, mis en ligne le 01 septembre 2010, consulté le 10 décembre 2020. URL http://journals.openedition.org/lapurdum/1896 ; DOI : https://doi.org/10.4000/lapurdum.1896 


\section{INSTITUTIONS, SOCIÉTÉ ET NOBLESSE EN PAYS BASQUE : QUELQUES TEXTES SIGNIFICATIFS}

\section{Présentation générale :}

Il manque toujours une histoire générale de la noblesse en Pays basque Nord ${ }^{(1)}$. Lors du Deuxième Congrès Mondial Basque, J.-B. Orpustan terminait sa communication sur "La noblesse rurale dans la Basse-Navarre médiévale" par une remarque pertinente :

"Vouloir définir la noblesse médiévale en Basse-Navarre revient à traiter l'une des questions centrales de la société basque ancienne (...) il faut souhaiter qu'un historien du Moyen Àge puisse mener plus loin et compléter les analyses qui ont été présentées ici" (2).

Malgré les importantes contributions de Jean de Bertier ${ }^{(3)}$, les travaux de recherche manquent pour connaître les patrimoines nobiliaires que pouvaient constituer les revenus agricoles, les dîmes, la gestion des forges, les produits des moulins, les transactions portant sur les terres nobles et conservées par les contrats de notaires, les revenus tirés de la carrière dans les armées du roi de France et de Navarre ${ }^{(4)}$ etc.

Pour les historiens, c'est un lieu commun de dire que l'exploitation des sources sérielles reste la méthode préconisée pour l'approche des réalités historiques. Mais des textes significatifs peuvent aussi apporter un éclairage précieux sur les situations d'affrontements ou de contestations que pouvait éventuellement engendrer la tutelle nobiliaire. Les trois documents que nous présentons touchent les domaines institutionnel, social, économique, à des moments différents de l'histoire des temps modernes. Sans doute ne faut-il demander au document original que ce qu'il peut nous donner et cela exige-t-il de multiplier les investigations en vue de constituer des séries. Ce travail reste à faire. En attendant, dans leur état actuel, ces documents posent plus d'une question sur le passé dans un Pays basque qui n'était pas bucolique tous les jours.

\section{1580 : La rivalité de deux seigneurs à l'Assemblée du pays de Cize.}

La scène se passe en 1580 dans le royaume de (Basse) Navarre. Henri III de Navarre a 27 ans. Il a fait du château de Nérac le centre de 
son gouvernement. Sa mère Jeanne est morte depuis huit ans ${ }^{(5)}$. En ce mois de novembre 1580, Henri de Navarre séjourne au château de SaintBrice entre Cognac et Jarnac. Il vient de signer le 26 novembre, la veille donc des événements relatés dans le document présenté ici, le traité de Fleix qui mit fin à la septième guerre de religion.

En cette année 1580, Arnaud de Gontaud, seigneur de SaintGeniès et d'Audoux, est président des États de Navarre "loctenent de sa Majestat", "représentant sa persone et de Madame sa sor" ${ }^{(6)}$ : la régence en Navarre était donc assurée par sa sœur Madame Catherine. Le "secrétaire du roi pour la convocation des États de Navarre" est Arnaud de Saint-Pic ${ }^{(7)}$.

La consultation des registres de la Chambre des Comptes permet de relever parmi les conseillers de la chancellerie les noms du Mixain Derdoi (est-ce le père de la future épouse d'Arnaud Oihenart?), du Souletin Enecot Sponde, ancien secrétaire de Jeanne d'Albret et nouveau secrétaire du roi Henri ${ }^{(8)}$, et l'un de ses fils, Jean, est étudiant à l'université de Bâle, "écolier entretenu" (9).

Depuis 1575, les États de Navarre réclament chaque année "l'observacion de la religion catholique et romane", "exceptat los de la religion reformade" (10), ce qui laisse supposer la persistance d'un parti calviniste minoritaire au sein des États.

En 1563, le meurtre du seigneur Bertrand de Suhescun, conseiller calviniste de la chancellerie, "tué en plein jour sur un chemin public en la chancellerie de Saint-Jean" constitua le début sanglant de l'affrontement des factions. Ce premier drame eut un grand retentissement. Un procès fut intenté par ses enfants et "dix gentilhommes" (11). Après quelques années d'accalmie, du moins d'après les les sources d'archives, le parti catholique prit l'étendard de la révolte en 1567 sous l'impulsion de Charles de Luxe. La paix revint en 1570 avec la signature du traité de Saint-Germain-en-Laye ${ }^{(12)}$. Mais Charles de Luxe ne reçut le pardon et ne réintégra ses biens confisqués qu'en avril 1572 à la mort de la reine Jeanne ${ }^{(13)}$. Il inspira la chanson "Atharratze Jauregiko Anderia" à la suite de son remariage à 49 ans avec Jeanne de Jaurgain, demoiselle de 17 ans $^{(14)}$.

Lalanne est le nom gasconnisé de Larrea dont le château est sis à Ispoure. Au moment du soulèvement du parti catholique en 1567, les deux frères Lalanne restèrent fidèles à la reine de Navarre. Grâce à l'appui d'Enecot Sponde, Jean reçut en récompense la commanderiehôpital de Saint-Michel qu'auparavant "feu sieur d'Anchiondo tenait" ${ }^{(15)}$. Marc fut pourvu de la charge de châtelain de Saint-Jean-Piedde-Port en 1569 en remplacement de Jean d'Armendaritz qui avait été accusé d'être l'assassin de Bertrand de Suhescun (16). La consultation des registres de la Chambre des Comptes pour l'année 1580 laisse quelque peu perplexe. Trois lignes d'écriture comptable mentionnent trois sortes de gages pour "Marc Lalanne le jeune capitaine retenu", "Marc Lalanne l'ainé pensionnaire", et "Lalanne châtelain de St Johan"(17). 
Le souvenir des exactions commises par les deux factions au cours des années 1567-1570 n'était certainement pas effacé en 1580: nombreux saccages en Ostabarret et Mixe, incendie de Larceveau ${ }^{(18)}$. En 1632 encore, les États de Navarre rappelaient que "durant les troubles de la religion il y a eu des bruslements et saccagements des villes et parroisses du present royaume, mesmes des principales maisons comme il se voit par les masures quy y restent" (19).

Entre les deux factions rivales, s'interpose le représentant de "Sa Magestat" qui a reçu "commission dirigide per le Rey" pour que l'Assemblée du pays de Cize se déroule conformément au droit. Martin de Logras est "avocat et procureur général faisant les deux estats" à la Chancellerie de Navarre depuis $15744^{(20)}$. Habitant Saint-Jean-Pied-dePort, il possède la ferme des greffes de la Cour des jurats et du Commissariat des finances de Saint-Jean-Pied-de-Port, et du greffe de la Cour d'Arberoue ${ }^{(21)}$. Il est représentatif du personnel judiciaire et administratif dont l'ascension sociale est liée au service du roi. Au début du XVII' siècle, Logras figurait sur la liste des "maisons affranchies" ou "rémissionnées", c'est-à-dire bénéficiant de grâces et d'exemptions ${ }^{(22)}$. À la fin du XVII' ${ }^{c}$ siècle, les descendants accédèrent au rang de la noblesse par achat de la "maison noble d'Olhonce du lieu de Çaro" (23). Entre temps, la famille consolida sa fortune dans l'industrie minière ${ }^{(24)}$.

Quelle était la parenté de Martin de Logras avec le célèbre "Arnaud de Logras, prieur d'Utziat (...) auteur de poésies au XVI ${ }^{\circ}$ siècle ${ }^{(25) "}$ ?

Comment était transmise en Pays basque, d'une génération à l'autre, cette langue juridique gasconne qui bénéficia jusqu'au début du $\mathrm{XVII}^{\mathrm{e}}$ siècle d'un statut de langue officielle dans le royaume de Navarre? Quel fut le rôle de l'Académie d'Orthez ou du Collège de Guyenne à Bordeaux ?

Tout en reconnaissant qu'il est difficile de répondre à ces questions, nous constatons que les années 1560-1580 constituent une période charnière sur le plan littéraire : avec Jean de Leizarraga, Pey de Garros, Arnaud de Salette ou Logras, les langues "vulgaires" prennent du lustre.

L'intérêt de ce document est aussi d'apporter un éclairage pertinent sur le fonctionnement des institutions. Á l'instar de chacune des vallées, ou pays de Basse-Navarre, l'organisation administrative du pays de Cize appartenait à l'assemblée générale des habitants qui se réunissaient à Saint-Jean-le-Vieux pour traiter des "affaires communes". Le seigneur de Luxe, quoique Mixain, pouvait y assister en tant que seigneur d'Ahaxe. L'objet de la présente assemblée est de pourvoir à la charge d'alcade de Cize ou juge royal qui remplissait en outre certaines tâches administratives ou fiscales. Le document restitue la réalité de cette assemblée, lieu d'affrontements d'intérêts antagoniques, de factions nobliaires rivales. L'instance de régulation, le procureur du roi en l'occurrence, avait pour objectif d'obtenir des protagonistes de "pausar las armes" à l'entrée du périmètre de l'assemblée et de "siéger sans procéder à aucune voie de fait" ("sens procedir a aucunes vies de feyt"). 
Enfin, on peut se demander dans quelle mesure la sémantique révèle la manière dont était perçue la composition de cette institution : assemblée "des voisins, manants et habitants"? ou "des châtelains, gentilshommes, infançons, voisins, manants et habitants"? Le glissement sémantique est-il un simple exercice de style ? ou reflète-t-il la réalité d'une société encadrée par la hiérarchie nobiliaire ? La question reste posée.

Le ton solennel, exprimé par le pluriel dit de majesté, le style apparemment pompeux, l'emploi de certaines formules juridiques, peuvent prêter à sourire. Mais le document, même dans sa forme littéraire et juridique, est révélateur de l'émergence progressive de l'État de droit à travers la personne du roi, ici celui de Navarre, garant de la justice. Certes la société garde encore les traits de l'héritage médiéval (n'oublions pas l'âpreté des luttes entre factions nobiliaires), mais en même temps, s'affirme, à l'image de Martin de Logras, un personnel administratif de lettrés, de juristes, véhiculant une conception du pouvoir qui repose non sur la force mais sur la justice. Une nouvelle science politique ou juridique s'élabore, inspirée par un corps de doctrine que l'on devine en filigrane dans les propos de Martin de Logras, affirme l'autonomie de la législation émanant de l'État souverain dans sa juridiction. Mais est-ce trop demander au document que d'engager la réflexion dans ce sens?

\section{Notes sur la présentation du document I}

(1) Pour la Navarre, il faut signaler l'ouvrage remarquable publié par Eloisa Ramirez Vaquero : Solidaridades nobiliarias y conflictos politicos en Navarra, 1387-1414, Pampelune, Principe de Viana, 1990.

(2) Actes du Deuxième Congrès Mondial Basque, Section Histoire du Pays basque, Saint-Sébastien, Txertoa, 1988, I, p. 251-267.

(3) Jean de Bertier, "Les entrées des nobles aux États de Navarre", Bulletin de la Société des Sciences, Lettres et Arts de Bayonne, 1962, p. 13-81; 1963, p. 1-7. "La noblesse inféodée dans les Pyrénées (Béarn, Navarre, Soule)", Bulletin de l'Association Noblesse Française, n 161, 1979, p. 25-51. La revue Ekaina (années 1991-1992) a également publié des articles de Murielle d'Abbadie, sur "La noblesse en Basse-Navarre au XVIII" siècle".

(4) En ce qui concerne la Basse-Navarre et les privilèges d'ordre fiscal, juridique, politique attachés à la noblesse, voir $\mathrm{A}$. Destrée.

(5) Bibliographie consultée : Jean-Pierre Babelon, Henri IV, Paris, Fayard, 1982 ; Christian Desplat, Henri IV, Itinéraire d'enfance, de gloire et d'amour, Pau, J\&D, 1991.

(6) Arch. dép. Pyr.-Atl., série C.

(7) D'après l'indemnité qui lui est versée en 1581 (Arch. dép. Pyr. Atl., B 2539). Voir aussi Arch. nat., K $1233 \mathrm{n}^{\circ} 23$.

(8) Arch. dép. Pyr.-Atl., B 149, F 18-19 et B 2489.

(9) Arch. dép. Pyr.-Atl., B 2677, 2484, 2489.

(10) Arch. nat., K 1233, $n^{\circ} 18$ et 18 bis ; Arch. dép. Pyr.-Atl., C 1531 ; archives du Musée basque à Bayonne, fonds Daranatz B-I (J bis).

(11) ibid. 
(12) Une abondante historiographie existe sur ces événements. A. Ruble, Jeanne d'Albret et la guerre civile, Paris, Guillemin, 1897. V. Dubarat, Le protestantisme en Béam ou au Pays Basque, Pau, Vignancour, 1895. R. Ritter, "Jeanne d'Albret et la Réforme chez les Basques", Eusko-Jakintza, 1951, 1952.

(13) D'après Jean de Jaurgain, Revue historique du Béarn et de la Navarre, juillet 1882, et arch. dép. Pyr.-Atl. B 2172, Bib. nat., fonds français, 1553, f० 243.

(14) Jean de Jaurgain, “Quelques légendes poétiques du Pays Basque”, La tradition au Pays Basque, Bayonne, Elkar, 1994, p. 385-393.

(15) Correspondance d'Enecot Sponde à Jeanne de Navarre, publiée par A. Communay, Les Huguenots dans le Béarn et la Navarre, Paris, Champion, 1885.

(16) Arch. dép. Pyr.-Atl., B 2152. Voir aussi Bibl. Nat., collection Baluze, vol. 151. R. Ritter, op. cit, , année 1951, p. 195-196.

(17) Arch. dép. Pyr.-Atl., B 155, fo 22-26. Voir aussi Les Amis de la Vieille-Navarre, $1^{\text {er }}$ semestre 1991, p. 42-43.

(18) Arch. dép. Pyr.-Atl., B 2370.

(19) Arch. dép. Pyr.-Atl., C 1545.

(20) Arch. dép. Pyr.-Atl., série B 150, 1423, 2210.

(21) Arch. dép. Pyr.-Atl., B 1417 fo 2-4.

(22) Martín de Vizcay, Drecho de naturaleza, 1621.

(23) Arch. dép. Pyr.-Atl., C 1533, fo 284.

(24) En 1582, Martin de Logras et Enecot Sponde furent autorisés à des recherches de mines de fer, de plomb et de cuivre (d'après P. Haristoy, Recherches historiques sur le Pays Basque, p. 375, qui ne donne pas malheureusement la référence de la source d'archive).

(25) J.-B. Orpustan, Précis d'histoire littéraire basque, Izpegi, 1996, p. 37.

Document I : Arch. Nat., K 1233, $\mathbf{n}^{\circ} 20$

\section{Procès-verbal sur une assemblée du Pays de Cize à Saint-Jean-Pied-de-Port}

27.9 bre 1580

L'an mil cincq centz oeytante et lo vingt et septal jorn deu mes de novembre viron las sieys hores de maitin deud. jorn nous Martin de Logras avocat et procureur General deu Rey et Commissarry en aqueste portant dipputtat a effectuar et metter a degude execution lo contengut de notre commission dirigide per le Rey en sa Chancellerie en datte deu vingt et sieys deud. present mes cy alliguade partinz de la ville de St Palay en Companie de Bartholome Cortes Comendayre de la Comande de Aphat Ospital et de Pedro de Lesaca nostre servitor puynaler ${ }^{(n)}$ habitant en la present ville et conthinuant notre camin fins rencontre ab Pedro de Irissarry vesin de la ville de St Jehan et porter real en lo locq et parropie de $V_{i l l e n a b e^{(2)}}$ en Cize ab loquoal et ab led. susd. de Cortes et de Lesaca nous avons descender et retirar ${ }^{(3)}$ viron les detz hores de maitin deud. jorn pour prendre la reffection a lad. Comande Aphat Ospital qui es joeinent et près 
viron de ung gict de harquebouse deu locq et parropie de St Jehan le Vielh ont de toute ancyanettat se thienen lad. Cort et assemblade generalles deus vesins manans et habitants deu pays de Cize et estant rettirats en lad. Comande lod. de Irissarry depart deffents de guere a perbo une bone troupe de homes a chibal armats de diverses armes passant par lo endret ayat ${ }^{(4)}$ comunement harluseta qui es compres en lod. locq et parropie de St Jehan le Vielh et que le med. de Irissarry nous fy repport et entendut aquet sonts ${ }^{(5)}$ aucunement nous arrestar en lad. Comande partins de guere a pié acompainats deud. de Irissarry et estant arribats a l'endret et place ont lasd. Cort et assemblade deud. pays de Cize se fen assis enter lo temple, lad. maison de Sorhoto et Palacyda ${ }^{(0)}$ aqui trobant en se promenant Charles Sr et Baron de Lucxe, don Tristan son fray, assistits et accompainats deu Sr de Arroçaing, de ung nomat capitene Lucxe, de Sans Sr de Lacarry et de son filh primogenit, de Jehan Sr de Amorots, de Auger Sr plus ancyen de Biremont, de Tristan Sr Juen de Behasquen, los tres de miexs, de François Sr de La Salle de Horta ${ }^{(7)}$, deu Sr Juen de Lissagaray de binues $^{(8)}$ de Pes de Larrandi Cappitene et Baille deu pays de Ostabares et de Pierre de Iriart Capittene, Sr Juen de la Salle de Echeppare de Ibarrola, losqouale deu pays de Ostabares ${ }^{(9)}$, de Jehan de Echaus Cappitene, et deu filh prin et hereter de Licdiry ${ }^{(10)}$ de Lantabat, et ung nomat Martin baille de la ville d'Ostabat et baillet de crampe se disent deud. Sr de Lucxe, de Guilhen de Echobarne Sry deud. Sr de Lucxe de la ville de Garris, de ung nomat Pier de la Borguade de Aincye ${ }^{(t)}$, lostouts homes a chibal armats de lors espade et pistoles tenents lorsd. espade en lors scintes et las pistoles en lors maas et un e deus susd. de sieys ${ }^{(12)}$ homes armats chacun de lors pedrinailhe ${ }^{(13)}$ et les beden en lo susd. equipadge mesmes en losd. locq. et place destinade pour thenir las Corts et assemblade generalles deud. pays de Cize, et estant certiorats que les castellans, gentius homes inffançones, vesins, manans et habittans deud. pays en lod. jorn se deben assemblar en Cort generalle a estar per los procedit a la nomination de aucuns personadge pour exercyr l'officy de alcade et judge ordinary dud. pays vaccant, nous susd. Commissarry pour obbiar a touts incombenients qui poyren succedir mandant se asemblar losd. de Lucxe et autres de los Companie cy desus nomats et mencyonats so que fut et estants ainxi assemblats los remostrant que aucun de lod. ne debe ni pode ignorar mesme estants de la quallittat que oren ${ }^{(1+)}$ los edits feyts per les Reys predecessors de sa Magestat de bone memory et conffirmats per sad. Magestat pour lo bien et repaus de lors subgects contenents tres exprese prohibicion de se assemblar en congregation et assemblade ilicyte en port d'armes en semblants locxs publicx, mesme au temps qui cour ${ }^{(15)}$ et portats actes que se deben far en toute pats tranquillitat et simplicytat a l'honor de Diu et ediffication deud. poble et que so nonobstasnt nous los beden occularement en lo susd. equipadge et assemblade ilicyte que no pode succedir aucun bien sinon tout escandal a cause de que et pour la contrabention que fasen auxd. edicts, prohitius, nous en vertu de nostre ditte commission los fins comandement a touts et chacuns los susd. de se retirar pausar lorsd. armes et se conthenir en tout deber jus la obedience de sa Magestat et de sousd. édicts a pene de incorrer per chacun de los las penes declarades per aquets, lasquoalles aussy nous susd. Commissary affin no 
pretendasse ignorance los y declarants, disen que dequi no se debe assemblar en nombre plus haut de tres en armes a pene de lors vittes et conffiscation de lors biens et en se haber ainxi asemblats aqueres haben incorrud et encorre plus grandes si se poden inpausar sino obedissen en se retirant et pausant lors armes et tals comandaments et declarations los y reiterant per une dues et tres beguades, susque lod. Sr. de Lucxe se monstrant bien fachat en si medixs nous dixo et respondo que luy trobabe bien estranger nostres dits comandements d'autant son intention no ere de alterar ni inquiettar lo poble ni cometter aucune acte aude service de sa Magestat sinon de lo obedir en tout et per tout comme tres fidel subgect comme fere apparer per los effects toutes bets que la occasion se presentere pour lo servicy de Sa Magestat declarant que per le feyt deudit servicy aucun no lui metere lo pie davant ni lo passeren en boluntat et que si lui ere acompainat deus susd. aquets eren gentius homes sous parents et amicxs qui ordinairemant an acostumat de ly far thonor et plaser de lo thenir companie quoant arribade vers lodit pays de Cize et Lucx circunvesins et que per autres vegaudes fasen lo medicxs biadge ly haben feyt medixs companie et que tant lui en son temps que sous predecessors en. lo loc an touts jorns acostumat de marchar en si $i^{(16)}$ et plus grande Companie que la susd. non solement en lod. pays de Cize mes en touts autres parts, mesmes quoant son anats far la obedience a sad. Magestat ont que sien estade et que no pode creder que lo Rey entendosse que ainxi fesse traitat, considerat mesme que lui no ere en lod. locq per autres fiis et intention que pour acystir en lad. Cort generalle comma Sr de Ahatze et en aquere advisar et balhar sa vots comma los autres suus la nomination deud. officy de alcade estant assettiat en lo locq et Siege que sous predecessors haben acostumat en los temps a settiar et lui en lo son sentz res alterar ny innobar comma habe feyt et pretende far Marcq de la Lane Castellan lo inquiettant en sous drets et perrogatibes susque et sus tout dixo aure lo recours a Sa Magestat per en haber declaration de sa boluntat et repparation deus grebis a lui infferits, de que et de tout nous offrin de far mention en nostre present procès-verabal en repportar aquet au Rey en sa Chancellerie, mes nonobstant tant a luy que aux autres de sa Companie los reyteraben los susdits commandemants une dues et tres veguades comme per avant a cause deus. reyterramant lod. de Lucxe se seppara ab los susdits de sa Comapnie et après haber palpat ${ }^{(17)}$ et confferit lo feyt $a b$ lod. nous benguo trobar et nous reytera los medix prespaux et responses que de sus tant a lui que aux de sad. Companie estant en tout so desus presents per testimoins mandats et requericts los. Pedro de Irissarry et Beinat de Urrispe demorant en laditte maison de Sorhoto aud. locq de St Jehan le Vielh, et ab tant nous departin deud. Locq.

Et après ung pettit interballe de temps entendut que le Poble se asemblabe per le feyt de lad. Court generalle et que lo Castellant ere aussy arribat $a b$ aucuns homes armats nous retornant a lad. place ont trobant viron de cent cinquoante homes vesins manans et habitans deud. Pays de Cize armats los ungs de lors espades, autres de lances et bordons et lod. Castellan accompainat de oeyt homes harquebousier a pié et de Domingo de Villenabe son loctenent touts losquoals ensemble lodit Senihor de 
Lucxe et tos de lad. Conpaine se retirant au distret et parquet ont lad. Court generalle se acostume thenir et aqui debaton longuement mesmes losd. de Lucxe et Castellan suus lors perroguatibes forme et possession de se asettiar sens procedir a aucunes vies de feyt avants modestements et de lors dires et altercats fins rethenir acte a meste Bertrand de Irigaray notari real aqui present loquoal acte sine ${ }^{(18)}$ coppie dequet es cy alliguat a cause de que deu contengut en aquet no es feyte aqui aucune mention et dautant lost touts susdits se departin sents procedir à la tenence de lad. Cort generalle requerit avons susd. Commissarry de enfar repport per y estar provebit affin la Justicy ordinary no bacqui per deffecte de Alcade et lo bien public no pattisque me offrin de so far et los mandant de biber en toute union et tranquillitat juus la obedience deu Rey et de sous édicts sents y contrabenir nous rettirens a lad. Ville de St Johan et nostre maison de Chaharrena en laquoalle haben demorat los vingt et oeyt et vingt et nabieme jorns deus. present mes at:enden que lodit de Irigaray remetosse lodit acte a nostre poder, loquoal ainxi que nous a dicts a differit si longuement a cause que tant lod. Castellan que los dipputtats deud. pais bolen beder le contengut en aquet davant de nous lo delivrar et no les y poden en sy breu temps los comunicar et lo trental et darres deud. mes font de retorn en la presente ville haben rettirat losd. acte deudit de Irigarray, de que et de tout fen veritader repport en testimoniadge dequero et de la veritat nous en soubs signats. Ainxi signat M. De Logras Comissarri susdit, bailhade per copie per my. Signé. de Logras Comisari susd.

Traduction du document I : Arch. Nat., K 1233, $n^{\circ} 20$

27 novembre 1580

Le vingt-sept novembre de l'an mil cinq cent quatre-vingt environ vers les six heures du matin, nous, Martin de Logras, avocat et procureur général du roi et commissaire député pour effectuer et mettre à exécution l'objet de la commission que le Roi nous confia en sa chancellerie le vingt-six du présent mois, étant parti de la ville de SaintPalais, en compagnie de Barthélémy Cortes, commandataire de la commanderie d'Aphat Ospital et de Pierre de Lesaca notre serviteur armé(1) habitant la présente ville, continuant notre chemin, rencontrâmes Pierre de Irissarri voisin de la ville de Saint-Jean, huissier royal du lieu et de la paroisse de Villeneuve ${ }^{(2)}$ en Cize. Accompagné de ce dernier ainsi que de Cortes et de Lesaca, nous nous sommes arrêté( ${ }^{(3)}$ vers les dix heures du matin pour nous restaurer à la commanderie d'Aphat Ospital qui est située environ à un jet d'arquebuse du lieu et de la paroisse de Saint-Jean-le-Vieux où de toute ancienneté se tiennent ladite cour et assemblée générale des voisins, manants et habitants du Pays de Cize. Pendant que nous étions dans ladite commanderie, ledit de Irissarri aperçut vite une bonne troupe d'hommes à cheval armés d'armes diverses cheminant par l'endroit appelé ${ }^{(4)}$ communément Harluseta 
situé en lieu et paroisse de Saint-Jean-le-Vieux. Le même de Irisarri nous en référa et après l'avoir entendu, nous partîmes vite à pied, sans (5) nous arrêter en ladite commanderie, accompagné dudit de Irisarri. Etant arrivé au lieu et endroit où se tiennent lesdites cours et assemblées dudit pays de Cize entre le tertre, ladite maison de Sorhoto et de Palacyda ${ }^{(0)}$, ayant trouvé en train de se promener Charles sieur et baron de Luxe, Tristan son frère, assistés et accompagnés du Sieur d'Arrocaing, d'un nommé capitaine Luxe, de Sans, sieur de Lacarry, et de son fils aîné, de Jehan sieur d'Amorots, d'Auger sieur ancien de Biremont, Tristan sieur jeune de Béhasque, les trois (qui m'accompagnaient), François sieur de la Salle de Hozta ${ }^{(7)}$, sieur jeune de Lisagarai de Biunez ${ }^{(8)}$, Pes de Larrandi capitaine et baile du pays d'Ostabarret, Pierre d'Iriart capitaine sieur jeune de la salle de Echeppare de Ibarrola, lesquels (sont) du pays d'Ostabarret ${ }^{(9)}$, Jehan de Echaus capitaine, le fils aîné héritier de Liceiry de Lantabat, un nommé Martin baile de la ville d'Ostabat, et se disant valet de chambre dudit sieur de Luxe, de Guillaume (Gilen) de Echobarne secrétaire dudit sieur de Luxe, de la ville de Garris, un nommé Pierre du bourg d'Aincye ${ }^{(11)}$, tous hommes à cheval, armés de leurs épées et de leurs pistolets, tenant lesdites épées à la ceinture, et les pistolets à la main, quelques-uns de ses susdits hommes ${ }^{(12)}$ revêtus de cottes de mailles ${ }^{(13)}$. Les voyant dans cet équipement dans le lieu et l'endroit destiné à la tenue des cours et assemblée générale dudit pays de Cize, et sachant que les châtelains, infançons, voisins, manants et habitants dudit pays devaient aujourd'hui s'assembler en cour générale pour procéder à la nomination de celui qui exercera la charge d'alcade et juge ordinaire dudit pays restée vacante, nous, commissaire, pour obvier à tous les inconvénients qui pourraient advenir, mandant auxdits Luxe et autres de la compagnie ci-dessus nommés et désignés de s'assembler, et une fois que cela fut fait, leur signifiant qu'aucun d'eux ne devait ni ne pouvait ignorer ${ }^{(14)}$, vu leur qualité les édits des rois prédécesseurs de Sa Majesté, de bonne mémoire, et confirmés par sadite Majesté pour le bien et le repos de leurs sujets, faisant expresse défense de s'assembler et de se réunir en manière illicite avec port d'armes en de tels lieux publics, même par les temps actuels ${ }^{(15)}$, et qu'ils devaient assurer en tout, paix, tranquillité et modération, pour l'honneur de Dieu et l'édification dudit peuple et que néanmoins nous les voyions de visu dans ledit équipement et en assemblée illicite dont il ne pouvait advenir aucun bien mais plutôt toutes sortes de malheurs car ils allaient à l'encontre desdits édits et défenses, nous en vertu de notre dite commission, fîmes commandement à tous et à chacun des susdits de se retirer, en tout devoir, dans l'obéissance à sa Majesté et à ses édits sous peine d'encourir, chacun, les peines prévues. En outre, pour qu'ils ne prétendissent pas ignorer, nous leur signifiâmes qu'ils ne devaient pas s'assembler plus de trois en armes sous peine de vie et confiscation de leurs biens, et que s'étant ainsi réunis, ils les avaient encourus et même plus si c'était possible, à moins qu'ils n'obéissent en se retirant et en déposant les armes. Et leur réitérant lesdits commandements et déclarations, une, deux, trois fois, sur quoi, ledit sieur de Luxe se montrant bien fâché, nous dit et répondit qu'il trouvait bien 
étrange notre dit commandement d'autant que son intention n'était pas d'altérer ni d'inquiéter le peuple, ni de commettre une quelconque action au service de Sa Majesté, sinon de lui obéir en tout et partout comme très fidèle sujet comme il le ferait apparaître en réalité chaque fois que l'occasion se présenterait pour le service de Sa Majesté, déclarant que pour ledit service personne n'oserait avancer d'un pied ni outrepasser et que s'il était accompagné des susdits gentilshommes ses parents et amis qui ordinairement ont coutume de lui faire l'honneur et le plaisir de lui tenir compagnie lorsqu'il vient vers ledit pays de Cize et lieux circonvoisins et que d'autres fois, faisant le même voyage, ils l'avaient fait en même compagnie et que aussi bien lui en son temps que ses prédécesseurs en le leur, ont toujours eu pour coutume de marcher ainsi, et en plus grande compagnie, non seulement dans le dit pays de Cize mais ailleurs et même quand ils sont allés faire soumission à Sa Majesté où qu'elle se fût trouvée, et qu'il ne pouvait croire que le Roi le traitât ainsi étant donné qu'il n'avait d'autre intention dans ledit lieu que d'assister à la cour générale comme sieur d'Ahaxe pour y opiner et donner sa voix comme les autres afin de pourvoir l'office d'alcade siégeant en lieu et place où ses prédécesseurs le faisaient de coutume quand il le fallait, sans rien altérer ni innover comme l'avait fait et prétendu faire Marc Lalanne châtelain qui l'inquiétait dans ses droits et prérogatives, sur quoi il dit qu'il aurait recours par devers Sa Majesté pour avoir déclaration de sa volonté et (obtenir) réparation des dommages subis, ce que nous proposâmes de mentionner dans notre procès-verbal, rapporté au Roi en sa chancellerie, mais néanmoins nous réitérâmes tant à lui qu'aux autres de sa compagnie les susdits commandements, une, deux, trois fois comme avant, de sorte que le dit de Luxe se sépara des autres (membres) de sa compagnie, et après avoir touché au doigt ${ }^{(17)}$ et conféré, il vint nous trouver et réitéra les mêmes propos et réponses et nous demanda copie de notre dite commission et du présent procès-verbal, ce que nous lui accordâmes, lui faisant néanmoins à nouveau les mêmes commandements tant à lui qu'à ceux de sadite compagnie, étant témoins de tout ceci, mandés et requis, ledit Pierre de Irisarry et Beinat de Urrispe demeurant en ladite maison de Sorhoto audit lieu de Saint-Jean-le-Vieux et nous quittâmes ledit lieu.

Et après un petit intervalle de temps, entendu que le peuple tenait cour générale et que le châtelain était aussi arrivé avec des hommes armés, nous retournâmes à ladite place où se trouvaient environ cent cinquante hommes voisins, manants et habitants dudit pays de Cize, armés les uns de leur épée, les autres de lances et de bâtons. Ledit châtelain (était) accompagné de huit hommes arquebusiers à pied, de Dominique Villeneuve son lieutenant. Tous ensemble avec ceux de la ladite compagnie du seigneur de Luxe s'étant retirés au district et parquet où ladite cour générale se tient de coutume, les dits de Luxe et châtelain discutèrent longuement sur les prérogatives, manière, possession du siège, sans procéder à aucune voie de fait, et de leurs dires et propos nous fîmes dresser acte par maître Bertrand de Irigaray notaire royal, ici présent, acte que j'ai signé et (dont j'ai joint) copie ${ }^{(18)}$ car ici il 
n'est pas fait mention du contenu, d'autant que les susdits se séparèrent sans procéder à la tenue de ladite cour générale. Nous, susdit commissaire, avons requis de faire rapport pour le faire valoir afin que la justice ordinaire ne défaille pas par manque d'alcade et que le bien public n'en souffre, nous proposâmes de l'exercer et nous leur ordonnâmes de vivre en toute union et tranquillité sous l'obéissance du Roi et de ses édits, sans y contrevenir. Nous nous retirâmes à ladite ville de SaintJean, dans notre maison de Chaharrena où nous demeurâmes le vingthuit et le vingt-neuf dudit présent mois en attendant que ledit Irigaray remît ledit acte en notre pouvoir, lequel ainsi qu'il nous l'a dit, a différé bien longtemps, car aussi bien le châtelain que lesdits députés dudit pays voulaient voir le contenu de l'acte avant de nous le délivrer, et ils ne le pouvaient pas en un temps si bref; et le trente-et-un dudit mois, de retour à la présente ville, ayant retiré ledit acte dudit de Irigaray, nous, soussigné en fîmes rapport véridique de tout ce que nous avions vu. Ainsi signé M. de Logras commissaire susdit, baillé pour copie par moi. Signé M. de Logras commissaire susdit.

\section{Document I :}

\section{Notes, remarques de transcription et de traduction}

(1). Ce terme n'est pas signalé par les différents dictionnaires historiques consultés. La traduction proposée ici le rapproche de l'espagnol puñal, "poignard", que le basque a aussi emprunté.

(2) Il s'agit de l'actuel village d'Iriberry.

(3) La lecture de ce passage fait problème dans la mesure où descender et retirar sont deux infinitifs. Manquerait-il un mot? À remarquer aussi que la forme est manifestement française.

(4) La forme ayat reste ici un mystère. La seule hypothèse est qu'il s'agit d'une abréviation de aperat.

(5) La forme surprend : on attendrait plutot sentz.

(6) Ce toponyme reste assez étrange : est-ce un diminutif de palaix "petit château"?

(7) Est-ce une transcription défectueuse de Hozta ? Dans ce cas, il s'agit de la maison noble de Hozta/Hosta mentionnée dans de nombreux documents médiévaux et modernes.

(8) Étrange toponyme : sans doute faut-il lire Bunus où est effectivement située la maison noble Elizagarai.

(9) La graphie Ostabares désigne en principe un pluriel (bares comme équivalent de valles) auquel il est difficile de donner un fondement logique.

(10) Sans doute faut-il lire Elizeiri, ancienne maison infançonne de Lantabat.

(11) Il s'agit soit d'Aincille, soit d'Ainhice, les deux villages étant situés en Cize.

(12) L'ensemble du passage fait problème : sans doute faut-il lire sieys comme adjectif possessif de la $3^{\circ}$ personne dont il existe une forme seiie diphtonguée en seiei. Mais susd. n'est pas non plus à sa place? Comment lire deus? Je propose : une deus susd. de sieys hommes, 'quelques-uns de ses dits hommes'. 
(13) Ce terme n'est pas signalé dans les dictionnaires historiques. Sans doute fautil le rapprocher de l'espagnol pretina, "ceinturon" : dans ce cas, le mot serait synonyme de "cotte de mailles". C'est la traduction préconisée ici.

(14) Cette forme pose problème, l'original du manuscrit ne permettant pas de lire eren, à moins d'opter pour le prétérit ancien de aver : agoren devenu oren par contraction.

(15) A noter la graphie francisante du verbe.

(16) Encore une graphie francisante.

(17) Il s'agit ici d'un terme juridique précis, paupa/palpar, utilisé dans les formules des libellés de jugement (voir dictionnaire de Lespy).

(18) Il est difficile de saisir la nature et le sens de ce mot. Nous avons opté pour une forme verbale : sine (pour signé ou sinhé), prétérit du verbe senhar/signar.

\section{Conflit entre les habitants d'Ostabat et le seigneur d'Uhart}

Voici un autre exemple où le despotisme de la tutelle nobiliaire suscite la véhémence des réactions de la communauté d'Ostabat. L'affrontement est encore plus évident entre les prétentions d'un noble et les prérogatives de la population. Les doléances que les habitants d'Ostabat portent à l'encontre du baron d'Uhart devant le Parlement de Navarre sont de plusieurs sortes : non-observation de la préséance dont ceux d'Ostabat estiment bénéficier à l'Assemblée du pays d'Ostabarret ainsi qu'aux fêtes patronales, abus de pouvoir, chantage et pression que le baron d'Uhart semble exercer à l'encontre des délégués d'Ostabat.

Au-delà des aspects politiques et juridiques du conflit qui oppose noblesse et population, le document apporte un éclairage précieux sur le plan de l'ethnologie et de l'économie.

Tout d'abord, la relation détaillée est du plus haut intérêt pour la connaissance de la pratique de la danse : lien avec les fêtes patronales et les célébrations liturgiques, aspect civil et social, importance de la première main de la première danse (le "roi de la fête"). Le conflit presque sanglant entre les jeunes d'Ostabat et de Juxue d'une part, le baron d'Uhart d'autre part, révèle que la danse est ici une cérémonie réglée, l'expression d'une organisation communautaire, voire d'un statut social. L'acte est exécuté selon un ordre, un rang social, exprime des valeurs qui ne sauraient être transgressées. La rituelle chorégraphique est affirmation sociale et politique ${ }^{(1)}$. Ce document d'archive conforte les études de Jean-Michel Guilcher sur les caractéristiques de "la danse solennelle d'ouverture de la fête conduite par le premier magistrat", où "les participants prennent place dans la chaîne par rang d'ancienneté des maisons" (2). Et en terre d'Ostabarret, la présence de la maison Uhart ne pouvait être que récente par rapport à des coutumes et des règles rituelles anciennes.

Les prétentions du baron d'Uhart sur le "droit de paccage aux montaignes et herems d'Ostabarret", prétentions abusives selon les habi- 
tants d'Ostabat, laissent entrevoir l'aspect économique du conflit qui touche ici la gestion des terres communes. L'usage théoriquement collectif des biens du territoire ne veut pas dire que dans la pratique il n'y avait pas de situation de hiérarchie, d'inégalité, d'accaparement, de prééminence. Le baron d'Uhart a les moyens de faire paître dans les terres communes d'Ostabarret le bétail de Mixe et de Soule. Comment ? Le texte reste muet. Mais l'hypothèse la plus vraisemblable est le contrat de gasaille ou bail à cheptel. La pratique ne pouvait que renforcer la prééminence économique du baron d'Uhart, d'une part vis-à-vis de Mixains qui lui confiaient le troupeau moyennant des gains notables obtenus par les produits laitiers, le fumier, la laine ou même une partie du cheptel, d'autre part vis-à-vis des habitants d'Ostabat dont les possibilités du même espace pastoral communautaire étaient réduites par voie de conséquence. Sans doute le baron d'Uhart allait-il à l'encontre de coutumes "immémoriales", mais il ne transgressait aucun article écrit du For moderne de Basse-Navarre ${ }^{(3)}$. Il jouissait en l'occurrence de la prééminence juridique et économique.

En fait, ce document met à jour les comportements sociaux d'une puissante maison nobliaire. Dans cette fin du XVII ${ }^{e}$ siècle, le baron Clément d'Uhart a pris pour épouse Louise de Montréal de Moneins, héritère d'une maison dont les biens s'étendent en Labourd, BasseNavarre et Soule. Son père, Jacques d'Uhart, avait également pris pour épouse Elisabeth de Montréal-d'Urthubie, fille de Tristan, seigneur de Saut (Hasparren), Domezain, baron de Moneins. Quoique la maison noble d'Uhart fût sise en Mixe, Jacques d'Uhart fut pourvu en 1633 de la charge de bailli d'Ostabarret ${ }^{(4)}$.

\section{Notes sur la présentation du document II :}

(1) voir Jean-Michel Guilcher, La tradition de danse en Béarn et Pays Basque franf̧ais, Paris, 1984, p. 46-51.

(2) J.M. Guilcher, op. cit., p. 51.

(3) Sur les conditions d'élaboration et le contenu de ce corpus juridique, voir Jean Goyhenetche, For et coutumes de Basse-Navarre, Bayonne, Elkar, 1985.

(4) D’après Hubert Lamant, Armorial de Bayonne, Pays Basque et Sud Gascogne, T. I, p. 352-353.

\section{Document II :}

\section{Archives Départementales des Pyrénées-Atlantiques, 1J 895}

13 mai 1698. Requête du bailli et jurats d'Ostabat, demandant au Parlement de Pau de maintenir leurs privilèges et préséances contre les empiètements de messire Clément, baron d'Uhart. 


\section{A nos Seigneurs du Parlement.}

Suplient humblement les bailif et jurats de la ville d'Ostabat en Navarre disant qu'ils ont droit d'assister à toutes les cours générales qui se tiennent au pais d'Ostabaret par leur procureur ou députté, lequel donne en toutes les détibérations qui s'y prennent son sufrage ou opinion le premier. Il est aussi le premier nommé dans les autres actes qu'on passe concernant les affaires communes du pays d'Ostabares et lorsqu'il y a quelque solemnitté de messe nouvelle aud. pais, les habitants de lad. ville d'Ostabat vont à l'offrande avant les habitants des autres communautés dud. pais. Enfin il y a une solemnité le mois d'aoust de chaque année au lieu de Cibits, siège de la Cour Généralle, où après la messe on va à la danse et c'est un des habitans d'Ostabat qui a toujours accostumé de faire la première danse. Le Sieur baron d'Uhart jalous des droits et prerrogatives des supplians ne songe qu'à les leurs faire perdre. Il ne veut pas que le députté d'Ostabat opine en toutes les délibérations qui se prenent ausd. Cours Generalles, il les gronde et les menace, lesd. délibérations ne sont pas mesme dressées et signées sur le champ par les Sieurs magistrats et députtés qui scavent écrire, d'où il arrive mille inconvéniens et des surprinses manifestes. Il ne veut pas sortir du siège lorsqu'il s'agit de délibérer sur les affaires qui les regardent, et par sa présence il empêche qu'on n'y délibère à son désadvantage et s'il y a quelqu'un qui ne parle pas à son gré, il est méprisé et menacé d'être maltraitté. D'où il est arrivé que plusieurs magistrats ne veulent plus fréquenter lesd. Cours généralles, et les députtés n'y vont que parce qu'ils y sont constrains sous des peines. En telle sorte que les suplians sont obligés de se plaindre de tous ces procédés et de ce encore que led. Sieur bailif deffend le Secrétaire de recevoir des oppositions et apellations en la Cour de ses délibérations. Et il oblige par cest ordre les députtés à exécutter ses délibérations. Et enfin les suplians sont obligés de se plaindre de ce que le 28 aout dernier il se transporta dans la solemnité de Cibits avec des gens pour empêcher ceux d'Ostabat de faire la première danse. Il n'y eust à la vérité aucun d'Ostabat qu'y eut osé de s'entreprendre mais un jeune enfant de Juxue apellé Louis de Bordaberri ayant voulu mener lad. danse, led. Sr d'Uhart avec ses gens poursuivit ce garson l'espée à la main et on l'aurait mal traitté s'y led. Sieur d'Uhart par accident n'eust tombé par terre et que ses gens ne se feussent apliqués à le relever. C'est dans cette intervalle de temps que led. de Bordaberri se sauva. Après cella, led. Sieur d'Uhart fit faire la première danse à un particulier de Hosta et ne peut pas s'empêcher de dire que désormais il voulait régler la danse et ne souffrir pas que ceux d'Ostabat et de Juxue la fissent car (deux mots illisibles). Les députtés de ces deux communautés sont les seuls qui s'opposent à ses entreprises et qui ont voulu l'obliger à rendre compte des biens et revenus communs dont il est contable. C'est pour imprimer la peur aux habitans desd. communautés que led. Sieur d'Uhart se porta à faire cette violence qui est fort extraordinaire pour un homme de sa qualité et de s'estre prins à ce jeune garson qui ne lui faisoit nul tord. La première danse c'est à ceux de Juxue de la faire. Les fonctions dud. Sieur bailif sont limités par le for de Navarre qui ne lui donne d'autre droit que celui d'administrer la justice avec les autres magistrats, faire réparer 
les ponts et chemins et la taxe des vivres. Mais il veut mettre la main en toutes choses jusques la qu'il s'est avisé de prétendre qu'il a droit de paccage aux montaignes et herems communs d'Ostabaret pour ses bestiaux et pour ceux qui voudra y mener des pais de Soule et de Mixe ce qui est insoutenable parce qu'il n'a nul titre pour cella, au lieu que les suplians en ont pour faire voir que le bestail de ce pais est suiet au carnal. Ce considéré, il vous plaira de vos graces maintenir les suplians au droit d'assister ausd. Cours généralles par leur députté et à donner leur souffiage le premier en toutes les délibérations qui y seront prises et arrestées et aussi dans tous les actes qui seront passés concernant les affaires communes, et au droit d'aller aux messes nouvelles les premiers à l'offrande et avant les autres communautés dud. pais, et de faire la première danse à la solennité dud. lieu de Cibis, avec deffences aud. Sieur bailif et autres magistrats de les y troubler ordonner que led. Sieur bailif et autres magistrats suspects sortiront de l'assemblée en Cour Généralle lo"squ'il sera question de délibérer sur les affaires qui le regardent et laisseront les non suspects libres pour y délibérer, et que les desd. délibérations seront dressées et signées sur le champ, enjoindre au Secrétaire de recevoir tous actes d'opositions ou appellations, et au surplus faire inhibitions et deffences aud. Sieur d'Uhart d'introduire son bestail ni celuy du pais de Soulle et Mixe ausd. montaignes et herems à peine de carnal, et de mil livres d'amande avec despens, nomment pour leur procureur me Jean de Maseres et faire bien ainsy. Signé à l'original Detchemaite bailif et detchart jurat

La cour ordonne que les parties en viendront à l'audience avec les gens du Roy. Fait à Pau en Parlement, le Xe may 1698, Par la cour signé à l'orignal. Capot.

Le treisiesme may mil six cent quatre vingt dix huit au chasteau d'Uhart de la part des suplians signiffiée aud. Seigneur baron d'Uhart en personne qui a dit que tout ce qui est contenu dans la requeste qui lui vient d'estre signiffiée n'est qu'une continuation de faussettés calomnieuses et ingénieuses desd. Detchart et Detchemaite contre le Sr respondant, lesquels Detchart et Detchemaite se servent du nom des habitans d'Ostabaret (ligne illisible) Lesd. Detchart et Detchemaite doivent avant tout cuvre produire leur pouvoir et cindicat que lad. communauté leur aura donné sur ce sujet, après quoi led. Sr respondant offre de deffendre a lad. requeste, laquelle proprement parler n'est qu'un libel diffamatoire dont led. Sieur respondant demande réparation qui lui est deub en telle que la prudance de la Cour l'adjugera et a prins cette copie et signé. Fait par moi.

Balance, huissier, audr.

\section{Document III :}

\section{$\mathrm{XVIII}^{e}$ siècle : la question de la dîme en Pays de Cize}

Les registres des États de Navarre conservent des traces d'un différend qui surgit en Pays de Cize entre le tiers état et la noblesse de 1701 à 1783 au sujet de la date de versement de la dîme et des agneaux. 
Au vu des documents d'archives, il semble que la querelle s'apaisa à partir de 1784 .

On notera l'astuce des paysans de Cize, qui, sous des considérations d'ordre climatologique, réussissent à échapper en partie à la redevance du croît du bétail en avançant au maximum la date de vêlage, conformément au For moderne de Basse-Navarre, qui stipulait que la dîme des "chevreaux et agneaux" devait être perçue au premier mai. Retarder cette date jusqu'à fin juin signifiait pour le paysan-berger diminution du jeune cheptel et augmentation des besoins en "past".

L'utilisation des termes "dixme et prémices" laisse supposer qu'à la dîme du crô̂t du bétail s'ajoutait une charge perçue en argent ou en nature.

Ces documents jettent un éclairage pertinent sur les rapports sociaux au sein du monde rural en Basse-Navarre à lä fin de l'Ancien Régime. Peut-être aussi cette polémique est-elle révélatrice de la situation des campagnes à la même époque et de la conjoncture agricole. Car, au-delà de la contestation et de l'antagonisme noblesse-tiers état, la question est aussi de savoir si au XVIII ${ }^{e}$ siècle la dîme gardait encore son efficacité économique d'antan. Le comportement relationnel des décimateurs et des décimables ne s'est-il pas modifié en fonction de la conjoncture économique? Est-ce déjà le prélude au mouvement antidécimal que connut la France au printemps 1790 (décrets des 14 et 20 avril 1790 de l'Assemblée Nationale supprimant la dîme)? Nous ne pouvons que poser des questions : pour les élucider, l'enquête documentaire sur les contrats d'affermage durant une longue période serait d'un précieux secours.

Doc. III. 1. Année 1701 :

arch. dép. Pyr.-Atl., C 1533 f $^{\circ} 314$

Les Dixmes estant dues de droit divin chacun se devroit faire un point de religion de les paier avec exactitude et de manière que les ecclésiastiques quy les possèdent comme le véritable patrimoine de l'église ou les laïques qui les tiennent à titre d'inféodation en récompense des services rendeus à l'église en puissent profiter, celle des agneaux se doit payer suivant le for, titre des dixmes et prémices, rubrique 19, article 2 au premier de may, ce qui feut ainsy statué sans doute parce que du temps de la compilation du for on se conformoit encore à l'usage de la haute navarre et des pays de Soulle et de Labourt où les pasturages estant gras et abondans on donne encore aujourd'huy encore le bélier aux brebis de si bonne heure que les agneaux venant à naistre vers le commencement de l'hiver sont nourris et sevrés pour le premier de may, mais la sécheresse des terres de ce royaume estant telle que l'herbe n'y croist point du tout en hiver et que les brebis ne peuvent point estre assés nourries elles mesmes pour pouvoir bien nourrir des aigneaux dans cette saison, on a esté constraint de chercher quelque autre moyen de faire profiter le bétail à laisne, et on n'en a point trouvé de meilleur ny de plus utile que de donner le bellier plus tart 
aux brebis afin que les aigneaux ne venant que vers le printemps où l'herbe pousse, elles puissent nourrir leur fruit, ce quy fait que les aigneaux estant tardifs ils testent encore au premier de may et ne scauraient ny manger ni vivrez estant sevrés alors, de sorte que la loi faite à cet esgard par le for ne pouvant estre suivie sans rendre inutile et de nul fruit pour les décimateurs la dixme des aigneaux qui fait le principal revenu de leurs dixmes, il est d'une justice nécessaire et mesmes de l'utilité publique de la changer et d'étendre plus loin le temps du paiement de la dixme, ce changement se pouvant faire sur tout sans nul préjudice pour ceux quy tiennent des brebis, à ces causes il plaira Monseigneur à vostre grandeur ordonner que la dixme des aigneaux se paiera à l'avenir au premier de juin et que les décimateurs ny leurs fermiers ne seront pas obligés de les prendre plus tôt s'ils ne le veulent ainsy.

Led. Seigneur Lieutenant du Roy accorde aux supplians le contenu du présent article.

\section{Doc. III. 2. Année 1702 :}

\section{Arch. dép. Pyr.-Atl., C 1533, f $^{\circ} 321$}

Les nommés Minassagaray, Bisquay et Dechondo de Çaro sindics des habitants de Çaro, d'Uhart et Saint-Michel en Cize aiant présenté requête par devant monsieur le marquis de Lons lieutenant de Roy pour faire destruire le règlement prins par les Estats l'année dernière touchant le paiement de la dixme des aigneaux, Monsieur le Marquis de Lons a eu la bonté d'envoyer la requeste aux Estats pour y déliberer.

\section{Doc. III. 3. Année 1780 :}

\section{Arch. dép. Pyr.-Atl. C 1538, f 261-262 :} séance du 27 février

Dimes et prémices des agneaux.

Sur ce qui a été présenté que quoique par une disposition du For article 2, rubrique 19 des Dixmes et Prémices, l'époque du peyement des agneaux fut fixée au premier de may de chaque année, néanmoins les Etats reconnurent la nécessité de porter ce terme au premier de juin, à quoy il feut pourvu par règlement de 1701 homologué par arrêt de la Cour du 23 septembre même année. Cette détermination de la part des Etats eut pour objet d'empecher que cette espèce de dime ne devint inutille aux décimateurs ecclésiastiques et laiques qui en la recevant au terme réglé par la coutume, les agneaux étant encore trop jeunes venoient à périr, l'expérience aprend que ces mesmes inconvéniants se rencontrent en la payant suivant le réglement au premier juin. Dans cet état, il serait de la sagesse des Etats, par raport à la conservation et au repeuplement des bettes à laine dans le present royaume d'arretter qu'à l'avenir la dime ainsy que la prémice des agneaux ne sera payéque le vingt cinq juin, ouy le rapport des seigneurs commissaires, les Etats étant entrés en délibération, il ont esté par pluralité des suffrages de l'avis de la proposition, 
M. le sindic demeurant chargé de demander l'homologation de la présente délibération.

D'Etchebers, Prieur de Saint-Palais

D'Oihenart commissaire de la noblesse

Doc. III. 4. Année 1780 :

Arch. dép. Pyr.-Atl., C 1538, fo 271

Délibération provoquée par le tiers état au sujet des dîmes et prémices des agneaux.

Le tiers état a dit qu'il a été pris le 27 de ce mois une délibération concernant la dime et la prémice des agnaux, que le clergé et la noblesse ont délibéré que la livraison s'en ferait chaque année le 25 juin et le tiers état au contraire qu'il se feroit une suivant les réglements et usages des lieux et comme l'ordre du tiers état fait un interest sensible à cette perception, et à ce que l'usage des lieux ne soit point interverty, il propose qu'il soit de nouveau délibéré sur cette matière si intéressante. le clergé et la noblesse ont été d'avis qu'il n'y a pas lieu de délibérer sur ce, et le tiers état ayant délibéré, il a été de l'avis de la proposition, et ayant réopiné, il a été d'avis, vû la disposition du for, article 2, rubrique 19 des dîmes et prémices, qu'elle sera exécutée sans préjudice des usages locaux, et attendu la circonstance de l'opposition du clergé et de la noblesse en possession des dîmes et prémices, attendu aussi l'intérêt particulier du tiers état à maintenir les dispositions des fors et coutumes à ce sujet, il a nommé pour commissaire le sieur Lombart lieutenant de baillif et maire de Labastide Clairance avec pouvoir d'agir et se pourvoir dans cet objet par les voyes qu'il appartiendra, la noblesse a été pareillement d'avis de nommer M. Dechaux pour commissaire à l'effet de deffendre contre la réclamation du tiers état, et le clergé s'est abstenu de délibérer sur ce.

D'Etchebers prieur de Saint-Pclais, D'Oihenart, commissaire de la noblesse Lombart

Doc. III. 5. Année 1783 :

Arch. dép. Pyr.-Atl., C $1538 \mathrm{f}^{\circ} \mathbf{4 0 0}$

Dizmes et prémices des agneaux,

M. D'Echaux a dit qu'en exécution des deux délibération des Etats en datte des 27 et 29 février 1780 concernant les dixmes et prémices des agneaux, il s'est pourveu en la cour en sa qualité de commissaire de la noblesse et obtenu en contradiction deffenses avec le commissaire nommé par le Tiers Etat pour la cause, un arrêt qui renvoit les parties à se pourvoir par devers sa Majesté au fonds et au principal, et cependant ordonne l'exécution du Règlement de 1701 qui fixe l'époque du payement de cette prestation au premier juin de chaque année. De suite $M$. de Lombart ayant pris la parolle a dit qu'en 1780 étant dans l'ordre du Tiers Etat, il ne peut point refuser au témoignage de leur confiance d'accepter la mission de deffendre en cette cause, mais qu'ayant l'honneur d'être actuellement 
membre du corps de la noblesse il déclare à l'assemblée qu'il fait sa démission de sa qualité de commissaire pour ce regard.

Sur quoy les Etats étant entrés en délibération le clergé et la noblesse ont accueilli la démission de M. de Lombart et au surplus donné acte à M. D'Echaux de ses dilligences et le tiers état ayant persisté dans son arretté du 29 février 1780, vû la démission de M. de Lombart, a nommé à sa place le Sieur Delmas député de La Bastide Clarence.

D'Etchebers, Prieur Lombart

\section{Jean GOYHENETCHE}

Docteur en histoire

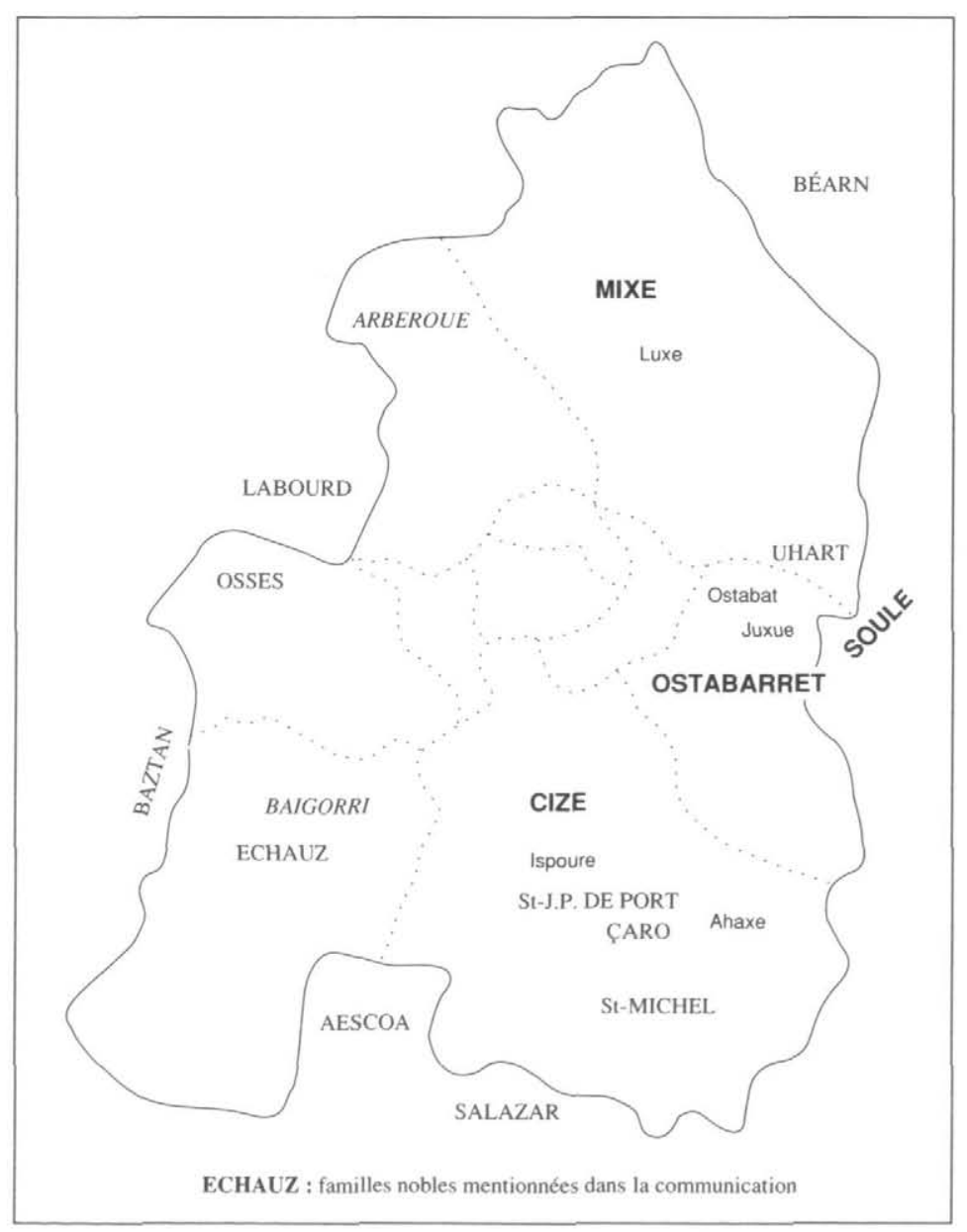

La Basse-Navarre et ses divisions territoriales. 